\title{
Research on the Influence of New Media Technology on Internet Short Video Content Production under Artificial Intelligence Background
}

\author{
Zhiqin Lu (iD) and Inyong Nam (iD) \\ Department of Mass Communication, Pukyong National University, Busan, Republic of Korea \\ Correspondence should be addressed to Inyong Nam; tarzan@pknu.ac.kr
}

Received 30 September 2020; Revised 25 December 2020; Accepted 14 January 2021; Published 28 January 2021

Academic Editor: Wei Wang

Copyright ( 2021 Zhiqin Lu and Inyong Nam. This is an open access article distributed under the Creative Commons Attribution License, which permits unrestricted use, distribution, and reproduction in any medium, provided the original work is properly cited.

\begin{abstract}
With the rapid development of the Internet and smart phone technology, a large number of short videos are shared through social platforms. Therefore, video content analysis is a very important and popular work in machine learning and artificial intelligence currently. However, it is very difficult to analyze all aspects of video content originally produced by large-scale users. How to screen out bad and illegal content from short videos published by a large number of users, select high-quality videos to share with other users, and improve the quality of video on the distribution platform of the entire user is a top priority. Based on this background, this paper focuses on optimizing video auditing to provide basic features for algorithm judgment, supporting original content and increasing the distribution of new content, and strengthening manual intervention combining algorithm recommendation with manual recommendation. Four major aspects of the artificial training algorithm model discuss the optimization effect of artificial intelligence on the algorithm in order to provide some guidance for the sustainable and healthy development of mobile short video.
\end{abstract}

\section{Introduction}

With the advent of the era of mobile Internet, people's access to information content is infinitely expanded, and the channel is infinitely convenient. As a new media form, short video is presented to users and has a huge user scale rapidly. Short video has a higher dimension of information presentation, and it is easier for people to accept and spread. With the gradual maturity of mobile Internet, cloud computing, artificial intelligence, and other technologies, the intelligent process of media is accelerating. The application of algorithms in news production has become a common trend. Major media organizations are actively developing the use of algorithms to promote the intelligent level of news production process. The application of artificial intelligence in new media communication is mainly reflected in machine writing and intelligent recommendation. The rapid development of media such as trembles, fast hands, volcano videos, and microblog videos has provided an effective platform for video transmission and sharing, making short videos the main carrier of multimedia transmission [1]. The user scale and video viewing duration are also growing day by day, becoming the mainstay of traffic on all major media platforms. In recent years, video data have exploded and more and more people like to show themselves by sending videos, attracting a large number of fans [2]. Short videos have become one of the most important and quick ways to become popular and even become a means of making a living. Therefore, more and more users publish small videos to publicize themselves or spread information. In the face of massive video, how to identify the content of short video, distinguish the quality of video, effectively recommend it to users, and how to observe hot events are crucial [3]. Users also hope to quickly find the videos that they are interested in, which are fresher, have a unique perspective, completely and coherently present scenes that are not often seen in life, and reduce unnecessary time expenditure. 
In recent years, depth learning has achieved great success in the field of images [4], and various international and domestic competitions have also spawned many excellent algorithms and models. On this basis, depth learning has been applied to many fields, including audio field [5]. Various deep networks developed on the basis of convolutional neural networks have greatly improved the video classification effect [6], fully demonstrating the important role and significance of convolutional neural networks in deep learning. Video content analysis requires a relatively complete understanding of the video content, which is to look at the video content from the perspective of a machine through a camera or video instead of understanding and analyzing the video content as a human identity area [7].

Besides, the concept of microvideo emerged in 2006. In 2013, "microvision" and "second shot" were launched. In 2014, "beauty shot" and "WeChat minivideo" appeared one after another. In 2016, the first year of short video broke out. By 2017, China Mobile's short video users had reached 242 million, and the short video capital market had accumulated more than 10 billion yuan [8]. Undoubtedly, mobile short video has become an important scene of Internet users' consumption in the era of artificial intelligence, of which development process and penetration have exceeded the original expectations and controls. It can be said that the significance of mobile short video has gone far beyond communication itself and has migrated to all fields and aspects of social life and has become a cultural, economic, social phenomenon, and existence, with subtle, lasting, and far-reaching impact [9]. From short video to mobile short video, the content is more and more dependent on the carrier; thus, the mobile short video is more closely combined with the recommendation algorithm. Convenient transmission, personalized information, and interactive consumption are the prominent features of mobile short video transmission, while fragmentation of content and upgrading of interest acquisition difficulty are the differences between mobile short video and long video and pictures and texts [10].

In the era of mobile Internet, the continuous expansion of mobile Internet users, the continuous improvement of the mobile communication network environment, the cultivation of users' video consumption habits, and the practical application of new technologies such as artificial intelligence have jointly given birth to the hot scene of social short video application in 2017 [11]. In 2016, a large number of mobile short video applications have appeared intensively, the capital market continued to heat up, the number of short video content entrepreneurs grown explosively, the short video industry ushered in a period of rapid development, and 2017 was called "the first year of short video" [12]. With the rapid development of social short video applications, a series of problems need to be studied and solved. With the maturity of tool attributes and the need of market competition, tool short videos begin to evolve into information and social short videos, and social short videos begin to differentiate from short videos as an independent classification. In this paper, short video, short video classification, and social short video are clearly defined, and the development history of social short video application at home and abroad is combed in detail. Through qualitative analysis, literature research, and case analysis, this paper analyzes the transmission mode and transmission effect of social short video application, taking "beautiful shooting," "fast hand," and "tremolo" as examples, and puts forward targeted optimization suggestions for transmission effect. All these provide theoretical support for the development of social short video industry and help the government to effectively control the emerging Internet form of short video, thus realizing the healthy and orderly development of domestic short video industry.

Based on the aforementioned background, this paper starts with the basic problems of mobile short video, summarizes the rise, concept, classification, and dissemination characteristics of mobile short video, and introduces its external appearance. Then, from the perspective of philosophy of technology, it applies the viewpoints of technology tool theory and technology value theory, mainly including technology self-enrichment, autonomy, subjectobject dualism, technology determinism, and environmental constructionism, from five aspects of algorithm logic, dissemination subject, dissemination content, audience, and dissemination effect.

\section{Definition of Short Video}

Academic circles and the industry have given different definitions on short video concepts, but there are several relatively mainstream definitions. "36 krypton" research institute defines it as "short video refers to video content with new media as the transmission channel and duration less than 5 minutes, which is another newly emerging content transmission carrier after text, pictures, and traditional video, and has the characteristics of low production cost, fast transmission speed, blurred boundaries between producers and consumers, and so on [13]." Figure 1 shows the software interface of tik tok, a short video software that is popular all over the world.

In addition, "I research consulting" defines it as "short video refers to the network video with a playing time of less than five minutes, which has the characteristics of strong social property, low creation threshold, short viewing time and convenient scene, and so on and is based on the video content form transmitted by PC and mobile terminals [14]." The following technology gives the definition of short video by comparing long videos: "the content produced by stars, Internet users, PGC, and UGC is the main content, and the duration is less than 5 minutes [15]." The duration of these short and medium videos is generally not more than 5 minutes. Academic circles have a relatively consistent view on the concept of short video, which is a new video form that can be shot, edited, and transmitted on mobile terminals such as mobile phones and other mobile terminals in units of seconds."

To sum up, the author believes that short video is a new form of video with length counted in seconds, mainly produced by stars, Internet celebrities, PGC, and UGC, and shot, edited, and processed by one key on mobile terminals 

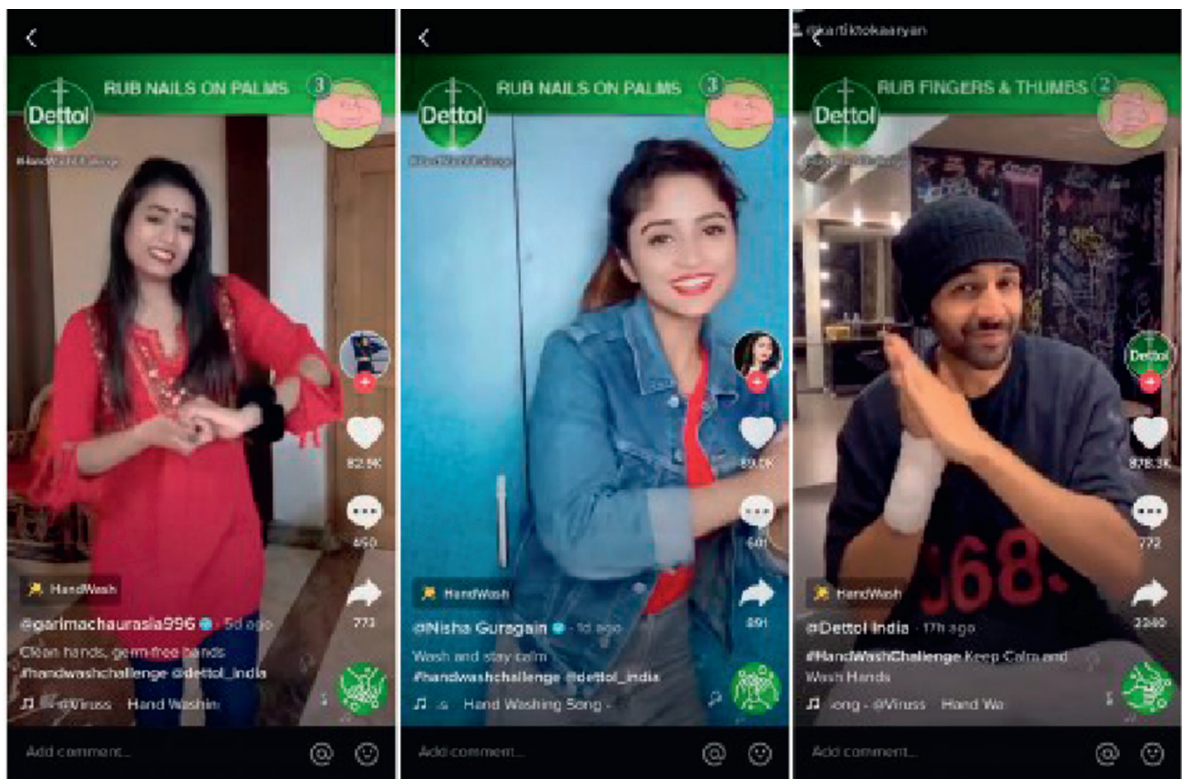

Figure 1: Short video software tik tok.

such as mobile phones and transmitted via external links, which has the characteristics of strong social attribute, low creation threshold, blurred boundaries between producers and consumers, and so on.

2.1. Classification of Short Video. There are different classification methods for short videos according to different standards, but in general there are mainly the following three classifications:

(1) Tools short video: Tool short video is a video production tool developed to meet the needs of personal production of short video, which has the functions of early shooting, editing, and processing and links its video content, such as small images and funny shots, through various communication channels [16]. Tool short videos tend to generate short-term high traffic in the early stage, but when users' enthusiasm wanes, the users and traffic accumulated in the early stage will also rapidly drain away. The transformation from photography tools to social videos is a matter that most mature tool short video companies are considering.

(2) Information short video: Information short video, also known as media short video, which is developed to meet the needs of users to find new things without shooting, beautifying video and social functions, such as volcanic small video and material. Information platforms are good at horizontal integration. Relying on high-traffic social or information platforms and providing short video playback functions, users will passively use such embedded short video support functions at high frequencies." There is also the problem of lack of social content, so forming a matrix horizontally with other social platforms and guiding each other is a great way for such platforms."
(3) Social short videos: Social short videos, also known as community short videos, are developed to meet users' social needs. They have high user stickiness and strong social atmosphere and are represented by fast hands, beautiful shots, and trembles. Looking at these three types of short videos, tool attributes are the most basic attributes of short videos. However, the feature of tool products that "only come when you use them and go when you use them" makes their users less sticky and easy to be imitated, causing them to begin to evolve towards information and social communication based on tool attributes in order to seek a longer-term development.

At present, there is no clear definition of the concept of "social short video app." The academic circles mostly refer to the concept of "mobile short video social application." Zhang Zixuan has simply defined the mobile short video social application, which is less than 30 seconds long and supports shooting, editing, and beautification on mobile terminals such as mobile phones [17]. Huang Weining explained the concept in more detail: "mobile short video social application is an intelligent mobile terminal-based application that integrates video shooting and social functions, which allows users to shoot a very short video and supports uploading it to the Internet after quick processing, directly linked seamlessly with a variety of online social platforms to realize social functions of sharing and interaction and a multimedia social communication channel integrating words, pictures, voice, and video [18]."

Based on the above analysis, the author defines the concept of social short video app, which is developed to meet the social needs of users, an application that can shoot videos, edit, and process videos, and spread through external links through mobile terminals such as mobile phones with high user stickiness and strong social atmosphere. 
2.2. The Reason Analysis of Short Video Rising. The soaring demand for fragmented information: In the era of the data explosion, people are producing data all the time. Facebook updates more than 10 million photos every day, and YouTube receives as many as 800 million visitors every month [19]. On average, videos are uploaded for more than one hour per second. The amount of information on Twitter almost doubles every year. More than 400 million microblogs are published every day, and these data are growing continuously. The speed of information explosion on social platforms is seriously out of balance with people's ability to accept and process information. The contradiction between the increasing amount of social content information and the serious lack of attention of users has led to a high demand for fragmented information. The short video clearly meets users' needs for fragmented information.

Social media demand for new forms of content: Whether the Twitter and Facebook abroad or WeChat and Weibo at home, traditional mobile social networking is declining, trying to find new forms to usher in the second explosive growth of social platforms. Short video has undoubtedly become a new development direction. Major mobile social companies are actively laying out their short video strategies. Twitter has launched Vine, Facebook has launched Instagram, and short video socializing has extended social scenes, increased social interest, and is gradually changing people's social habits and social life.

People's demand for personalized expression: Personalized expression is based on the uniqueness of life individuals. Individuality proves the existence of "self." In this era of high-speed social operation of the Internet, people oppose being squeezed and assimilated by the social environment and continue to seek to highlight the uniqueness of individuals. Social short video applications are designed to meet the needs of interaction between people. People want to express themselves in a better personalized way. Obviously, traditional text and picture forms can no longer meet the personalized expression needs of current users. Short video emerges as the times require, displaying the information users want to convey comprehensively and vividly.

Venture capital and advertiser drive: Compared with tools and media short video applications, social short video has higher commercial liquidity and return on investment. Social short video attracts a large influx of capital. At the same time, the video advertising budget of traditional TV media is also constantly focusing on mobile video, while the video advertising resources (movies, TV plays, variety shows, and so on) of the head minister are scarce. Social mobile short videos are favored by advertisers due to their high traffic, high load capacity, and long tail effect in transmission. The arrival of funds promotes the growth of the number of social short video platforms and encourages the output of more short video quality content.

\section{The Development of Short Video}

3.1. The Development of Short Video Abroad. Viddy is the earliest social short video application, which was released in the United States in 2011 [20]. Users can use it to capture memory, show off technology, and share new video content, which was once seen as the next Instagram, but due to poor management, it was annexed and acquired by YouTube. On January 24, 2013, Twitter released Vine, a short video sharing software that supports 6-second shooting, with automatic video splicing [21].

Vine quickly broke into the public eye, and the number of users had already broken the 40 million mark in 2013. Like Viddy, Vine eventually failed due to a large loss of important users. Instagram, which went public with Vine at the same time, developed in the social short video field. In 2013, the photo social application Instagram began to provide users with short video shooting function within 15 seconds. Based on Instagram's original large fan base, Instagram quickly achieved success in the social short video application field. After that, Instagram launched Hyperlapse, which has a new method of video duration compression and video character scene conversion, making short video applications no longer limited to the positioning of self-presentation.

At present, Instagram and Snapchat are the two largest social short video application markets at home and abroad. In 2012, Snapchat, a photo messaging mobile application selling "burn after reading," introduced a new version of the application that can record and send video messages. The newly introduced video messages can last up to 10 seconds and only allow recipients to watch them once in the application.

On August 15, 2017, the US technology blog TechCrunch reported that Snapchat is currently testing a new feature called Crowd Surf, which combines personal video clips from different users to create a seamless video effect lasting more than 10 seconds. Snapchat hopes to encourage users to continue short video social sharing through Crowd Surf. Snapchat and Instagram have both basic social functions and rich PGC + UGC content [22], which can be said to be a short video model of strong social and entertainment. Besides Snapchat and Instagram, musically and Mico have become the most promising social short video applications abroad [23].

3.2. The Development of Short Video. The embryonic period (2004-2011): The birth of short videos in the PC era Video services has already appeared, but the real breakthrough was the establishment of Letv in 2004, which represents the birth of professional video websites. Since then until 2011, major domestic professional video websites have been established one after another, and short videos in the PC era have been born. Short videos in the era of video sharing websites are only a supplementary form of long videos, with strong media attributes and weak social attributes. The emphasis is on the transmission of messages. Most of its contents are directly transporting high-quality foreign content or processing and adapting long videos.

Exploration period (2011-2015): All kinds of mobile short videos started. GIF Express went online in July 2011 and transformed into a short video community in March of the following year, which started almost at the same time as Snapchat, the most popular social short video application 
abroad. In 2013, the application of seconds for short information video was officially launched, and in the following year, new functions such as watermark and intelligent voice change were introduced. The play of short video was constantly updated. Meipai, an important product of Meitu Company, went online in May 2014 [24]. With the strategic goal of "girls' favorite short video community," Meitu's beautification application platform and intelligent hardware channels are backed up to achieve rapid exposure among target users. Meipai has acquired a large number of users through the initial star drainage strategy and then transformed from tools to communities through diversified operation methods. At the same time, Meipai has implemented the open platform strategy to form application ecology with other video and image applications, which has continuously explored the richness of functions, contents, and playing methods to form a more active user community. In general, from 2011 to 2015, with the popularization of mobile Internet and the formation of user habits in the era of fragmentation, all kinds of short mobile video products began to try out one after another, with platforms such as seconds, beauty, and fast hands gradually breaking through. Mobile phones began to become shooting tools, and users began to form the habit of watching short videos based on mobile terminals.

Growth period (2016): Mobile short video applications are available intensively. In July 2016, a short video was financed with RMB 100 million. The tremolo app went online in September, and the pear video went online in November. Throughout 2016, the number of short video app has exploded. Major Internet giants have laid out the short video field in an all-round way. Short video has become an investment outlet for a while, ushering in a period of rapid development in the short video industry. At this time, short video no longer appears as a supplement to long video, forming an obvious watershed with long video in content form, creator attribute, and operation mode.

Maturity period (2017-present): The social attribute of short video applications is increasingly prominent. In 2017, short video development has entered maturity. While subsidizing UGC content producers in a large amount, exploring commercial cashing mode has become an urgent problem to be solved for short video, which leads to the increasingly prominent social attribute of short video, because the commercialization of social products is more mature and abundant than that of tool products. For example, after the launch of the prop system in June 2016, users can comment on relevant content or interact with producers of short video content by giving virtual gifts, which directly brings about a direct increase in the user fees for the US auction in the current quarter. In May 2017, the daily average video broadcast volume of tremolo broke 100 million, while it reached 1 billion in August of the same year. Tremolo developed rapidly in a short period of time, and "suddenly appeared" in a number of short video applications, which has a great relationship with its social positioning of music. Zhong Danian, director of the China Network Video Research Center, said, "2017 is a wonderful year for short video bursts, which makes network video enter the most essential social attribute of the Internet-sociality [25]. As a new trend of the network phenomenon, video social communication has attracted the attention of the industry, academic circles, and investment circles. Table 1 lists current typical social short video app at home and abroad.

\section{Requirement Analysis of Short Video Content Analysis Algorithm Research}

For video and audio, manual audit usually needs to browse the video content completely or take the risk of missing audit for spot check, while artificial intelligence can analyze the whole content segment frame by frame at the same time node, mark, and deal with it accordingly. In the era of digital information explosion, the scale of data is enormous and even difficult to count every day. Data have penetrated into all walks of life and become an important factor of production. New media is the beneficiary of the era of "big data," and data are important advantage of new media. Data collection, data management, data analysis, and data utilization can be used by new media and generate considerable profits. Today's headline is a typical successful case of using the artificial intelligence algorithm [26]. It uses artificial intelligence to record audience's behaviors, classifies these behaviors, and draws a special chart for each audience. The more users' behaviors and characteristics, the more data collected by artificial intelligence and the more detailed charts drawn. In the interactive link of new media, the best comments are ranked through various algorithms, and the wisdom of the public is presented in the comment and post areas so as to better attract users to participate. In the stage of news recommendation and distribution, the types of news recommendation algorithms are evolving, and these new media intelligent recommendation algorithms based on the network are becoming new forms of power [27]. For the new media platform, although the audience's screen display position can slide infinitely to refresh new content, the priority order is limited, and the platform can calculate the most popular content by the algorithm and show it to the audience first.

In the microblog mobile phone terminal, the user can have two functions in short video. As shown in Figure 2, the top column of the microblog mobile phone terminal is a row of head portraits, and each head portrait represents a small video released by the user. User can view the short video released by the user by clicking on the head portraits of other users on the microblog platform. After the short video is played, the next short video will be automatically played. When the user pulls down and refreshes, the microblog client will update the new short video to the user to increase the user experience. Each user can also publish the video recorded or made by himself, share the video, and forward the video released by others.

There are three functions in the background of the system. Video released by users is processed through different depth learning models, including judging avatars through the open_nsfw model of porn, defining all avatars with more than 80 points as pornographic avatars, pulling 
TABLE 1: Analysis of present situation of typical social short video app at home and abroad.

\begin{tabular}{lcccc}
\hline $\begin{array}{l}\text { Type } \\
\text { Name }\end{array}$ & $\begin{array}{c}\text { Domestic } \\
\text { Quick worker }\end{array}$ & Beauty shot & Foreign \\
\hline Icon & Smile technology & Meitu net & Today's headlines & Facebook \\
Developers & 2011.3 & 2014.4 & 2016.9 & 2010.10 \\
Uptime & 17 seconds & 10 seconds & 15 seconds & 15 seconds \\
Video length & & & Snapchat \\
\hline
\end{tabular}

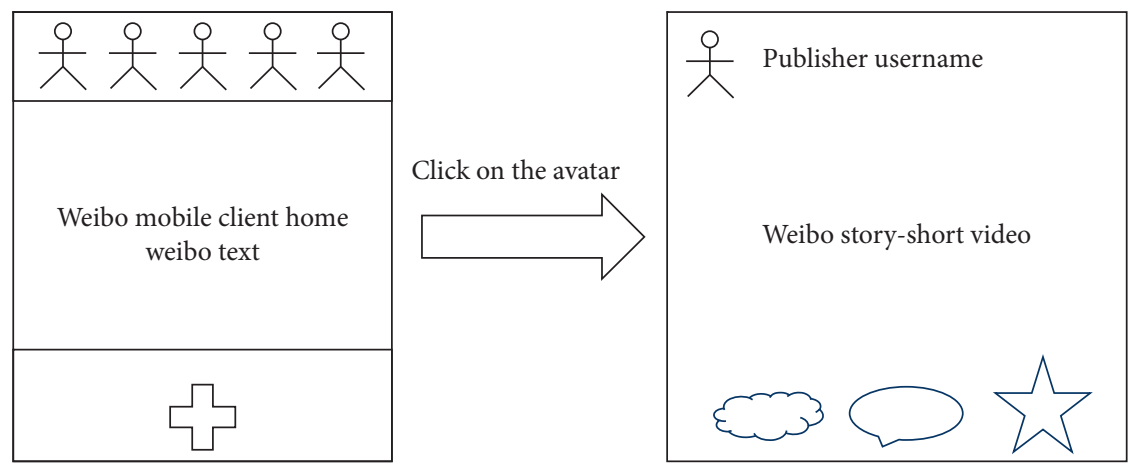

FIGURE 2: Interface diagram of microblog mobile phone client.

them into the blacklist, defining avatars with less than 80 points as nonpornographic, and continuing video content analysis. Video content discrimination firstly uses open_CV to extract frames, then uses the inception-v3 network model to extract feature vectors, and constructs multiple classifiers to classify after extraction. Three classifiers are constructed in this topic, namely, full connection classifier, support vector machine (SVM) classifier, and LSTM classifier, and the three classifiers are improved and optimized. Finally, experimental comparison shows that LSTM classifier achieves the best effect, and LSTM classifier is currently online. The third function is to use the improved vggish network model. First, ffmpeg is used to extract the audio from the video. Then, it is put into the depth learning model vggish. After online model classification, the audio is divided into three categories. Finally, the classified categories of audio are put into the video features to help video analysis.

4.1. Algorithm Architecture Design. The flow chart of algorithm architecture design is shown in Figure 3.

User data analysis involves many factors, so it is assumed that $x_{i} \in R^{n}$ is the factor influencing user data analysis and $y_{i}$ is the value of user data analysis. The user data analysis model is to find the relationship between $x_{i}$ and $y$ :

$$
\begin{aligned}
f: R^{n} & \longrightarrow R, \\
y_{i} & =f\left(x_{i}\right),
\end{aligned}
$$

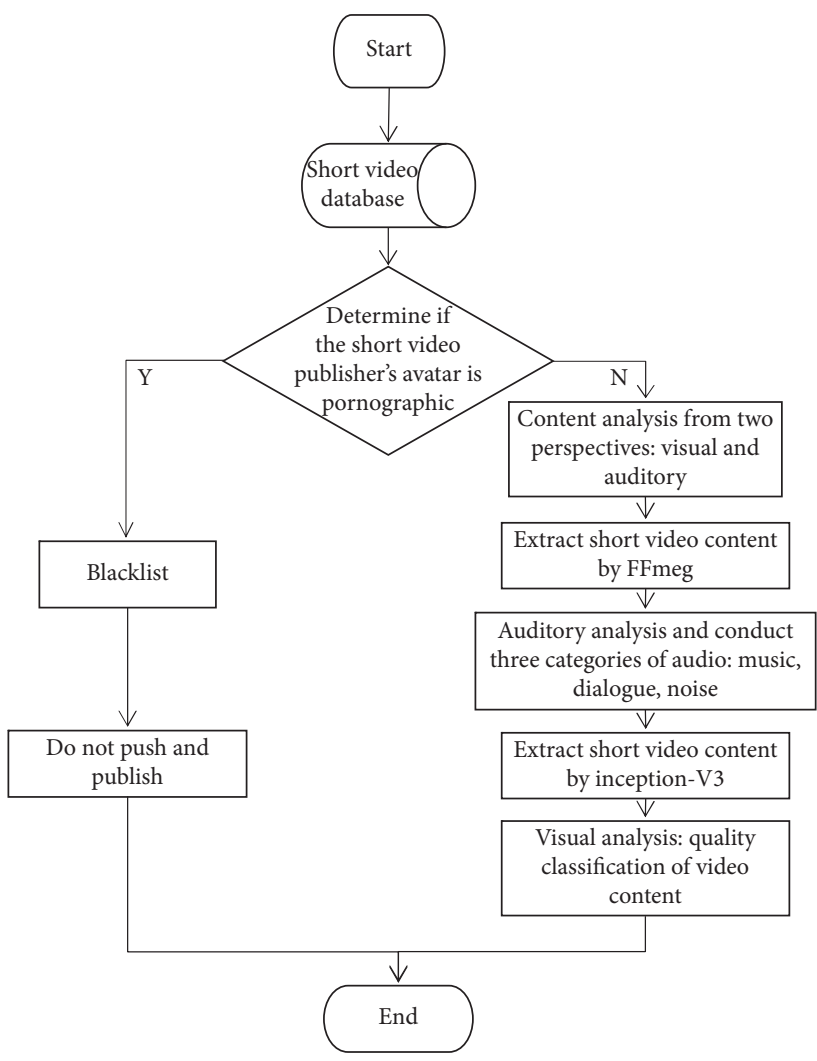

FIgUre 3: Flow chart of algorithm architecture design. 
where $R^{n}$ is a factor that affects user data analysis. According to SVM theory, the establishment of the user data analysis model seeks to establish the following expression:

$$
f(x)=\sum_{i=1}^{k}\left(a_{i}-a_{i}^{*}\right) K\left(x, x_{i}\right)+b,
$$

where $x$ is a factor that affects user data, $x_{i}$ is the $i$-th sample among $k$ samples, $K\left(x, x_{i}\right)$ is the kernel function, and the kernel function uses radial basis functions, as shown in the following formula:

$$
K(x, y)=\exp \left|-\frac{\|x-y\|^{2}}{2 \sigma^{2}}\right| .
$$

By studying the internal structural relationship of the index system, the problem of multiple indicators is changed into a multivariate statistical analysis method of a few indicators. A classic pattern recognition system based on statistical methods can include four parts: information acquisition, preprocessing, feature extraction and selection, and classification decision, as shown in Figure 4.

The management information base defines each data item that must be saved by the managed device and allows the operation and meaning of each data item. In network topology discovery, the variables of management information base that may be used are shown in Table 2 .

When the host where the algorithm runs is in the prediscovered subnet, the host is named as a flag node. The variation trend of target value with iteration times is shown in Figure 5.

After opening the microblog on the mobile phone, the user clicks on the head portrait in the top column of the microblog mobile phone client and sees a small video. Therefore, the analysis of short video content must first determine whether the user's avatar that publishes the short video is a pornographic avatar. If so, the uid of the user will be directly drawn into the blacklist, and other users cannot watch the videos published by users who enter the blacklist, because if a user's avatar is pornographic, the microblog content that he publishes is often pornographic, to prevent the dissemination of illegal content.

If it is a nonpornographic head portrait, it is necessary to judge whether the video content is of high quality. If it is of high quality, it can be added to the high-quality queue and pushed to the platform for other users. In the analysis of video content, because the video itself contains picture content and background music, the user also watches a video from both visual and auditory angles, so it is necessary to analyze the video picture content from both visual and auditory angles so that a video can be comprehensively judged and analyzed. The audio is extracted from the video by FFmpeg, analyzed and recognized, and a depth learning model is established to classify into three categories: music, noise, and dialogue. Then, the speech classification result is added to the video features as a feature to play a role in video classification.

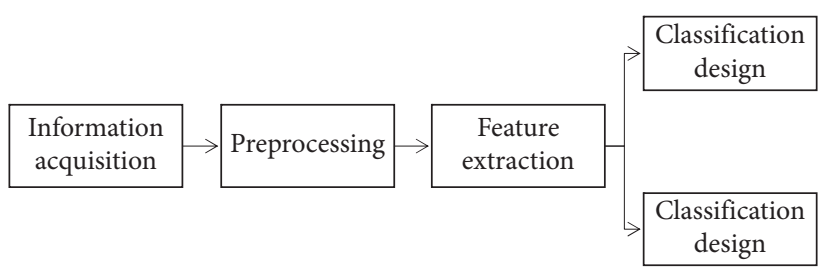

FIgURE 4: Basic structure of the model recognition system.

4.2. Functional Description of Algorithm Framework. In this section, the functional requirements and nonfunctional requirements of the system are described in detail in combination with the requirements use case diagram. The business use case diagram is shown in Figure 6.

According to the description in the section, it can be seen that this algorithm model is used by two people: one is the developer, the developer of this project, who want to complete the functions such as data set construction, network structure construction, and training; the other is the user of the model, who upload their avatar, check and verify the model, then publish the video they made, and judge through the model. From the user's point of view, the system requirements are as follows:

(1) Upload one's head portrait to the microblog platform: for the trained porn image computing network, the user must first upload his head portrait to see if his head portrait has been uploaded successfully

(2) Release the video produced by yourself: when releasing the video to see if it is released successfully and whether it is pushed to the microblog story sharing interface

For developers, the package can still be divided into three use cases from a large scope: pornographic avatar identification, video quality classification, and audio classification, including the following two use cases:

(1) Data set construction: extracting frames from videos uploaded by users and extracting features as network inputs.

(2) Training network: the training network includes six parts: data reading, network model construction, weight offset initialization, data forward propagation, error back propagation, and weight iterative training adjustment and model storage. Data reading refers to transferring training data to the input layer. Weight offset initialization refers to that full connection (FC) or support vector machine (SVM), or long-term and short-term memory (LSTM) network needs some initial weight offset values and the like as initial values to start training (generally, a value between 0 and 1 is randomly initialized). According to the different initialization methods used, the starting values are also different, and the initial 
TABLE 2: Management information base variable.

\begin{tabular}{lcc}
\hline Variable name & Variable attribute & Variable description \\
\hline sysName & 1.2 .1 .5 .1 .4 .1 .1 .3 & Device name \\
sysServices & 1.1 .2 .5 .2 .3 .2 .1 .3 & Service type \\
sysDescr & 1.3 .2 .5 .1 .3 .3 .2 .3 & Describe \\
Dot1dBasePortIfIndex & 1.3 .5 .2 .1 .17 .1 .4 .2 .1 & Port number \\
ifDescr & 1.4 .5 .2 .2 .1 .3 .2 .1 .2 & Name corresponding to port number \\
\hline
\end{tabular}

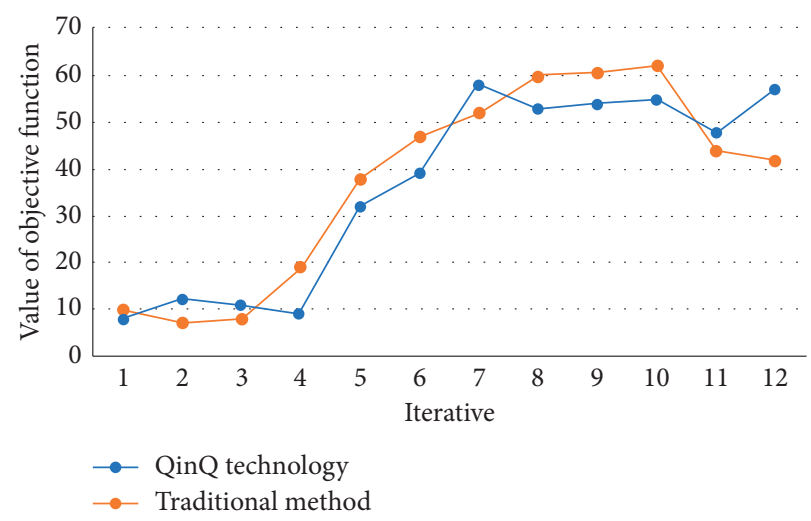

Figure 5: Change of target value with iteration times.

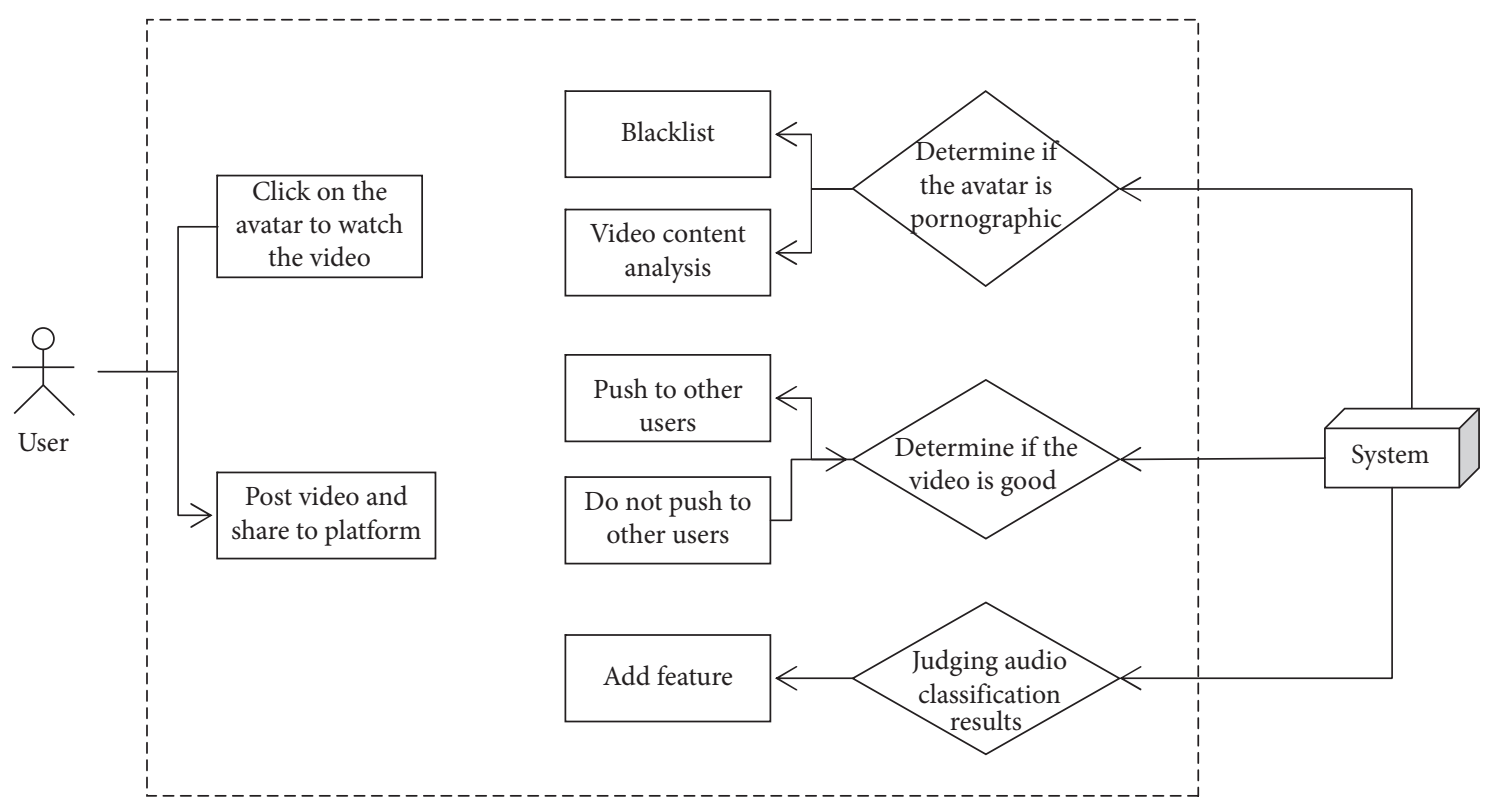

Figure 6: Business use case diagram.

values often greatly affect the final effect of the network model. The last four parts are necessary for training neural networks.

4.3. Nonfunctional Requirements of Algorithm Framework. It is not enough and incomplete for an excellent in-depth learning framework to have good function realization alone, which also has high performance requirements for itself, such as the pursuit of time performance. There are over 100 million daily users on microblogs, generating about $300,000 \mathrm{MB}$ of data sets every day. Faced with massive data, how to shorten training time better has become a very important problem [28]. A system must have strong robustness and practicability, which needs not only to realize functions but also strict requirements in terms of time efficiency, accuracy, training time and testing time, network generation efficiency, and classification and identification time. Therefore, the nonfunctional requirements of this system are as follows: 
(1) Network generation time: We have about 60 GB of video training data and hope to finish the training within 2 hours because if the training time is in progress, the training times will be reduced and the model cannot be debugged better. Debugging a model requires about 200 rounds of training and takes about two days.

(2) Classification training time: In the environment of CAFE caffe, tensorflow without GPU version, we have high requirements on training time. We hope to finish the training in as short a time as possible. Whether the training on CAFE of avatar image or the training on tensorlfow after video frame extraction, we hope to finish the classification training in 2 hours.

(3) Accuracy and recall rate: Because the final indexes of training are different, videos are the proportion of real high-quality videos that we hope to select. The head portrait classification hopes to kill by mistake rather than identify pornographic heads. Therefore, the head portrait classification is mainly TPR, with TPR reaching more than 96\%, while the video classification is mainly FPR and accuracy, with FPR reaching less than $1 \%$ and accuracy reaching more than $75 \%$.

(4) Test time: It is hoped that the test time will not exceed 10 minutes.

\section{Analysis on Propagation Mode of Short Video App}

The way of information acquisition, dissemination, and control in new media is empowered by technology. Intelligent algorithms and traditional information dissemination activities are integrated to form a brand-new information dissemination network. In a society where media and codes are everywhere, power is increasingly present in algorithms. Value judgment is based on people's values and subjective understanding, which evaluates and judges a specific object, and its judgment standard is generally the application value of the object to people. With the continuous optimization of media network information technology, many manual functions and powers in news dissemination, such as news clue discovery of journalists, content production, and news release of editors, are gradually transferred to algorithms, and the application scenarios and power scope of algorithms are expanding.

The dissemination of short video applications is different from traditional network dissemination, of which elements and process have their own particularity, which are mainly reflected in the following aspects: the dissemination subject has realized the transformation from "the recipient" to "the audience" role; the content information is very large and easy to spread with obvious attributes [29]. The ultrastrong timeliness of communication makes the interactive communication between users break the time and space limit, and the "newly occurred facts" and "media presented facts" can be presented almost synchronously.
5.1. Users of Short Video Applications. The advent of the mobile Internet era challenges the traditional mode of communication, and "users" have replaced the traditional "communicators" and "audiences." Users constantly change their roles between the two roles of "communicators" and "audiences," and what role they are in depends on what functions they use app. If they upload videos, they play the role of "communicators," and if they play videos, they play the role of "audiences." The "users" who play the role of "disseminators" mainly include ordinary users, platform professional users, and professional organization users, corresponding to UGC, PUGC, and PGC, respectively.

In the early stage of the development of short videos, the creators were mostly "individual retail investors," not professional individual producers. They only created and uploaded content based on individual interests, which is the content production mode of UGC. After the short video platform was put into operation for a period of time, the content producers began to differentiate, and those professional user producers with fan base or professional knowledge in a certain field began to differentiate from ordinary users, forming PUGC production mode. At the same time, there are some small groups specializing in production and production. They are usually independent of short video platforms, and their content production method is called PGC [30].

UGC is located at the bottom of the user pyramid due to its low cost and low threshold, which has a strong attribute and a large number of users. PUGC content is more valuable than UGC because it has accumulated a lot of popularity and traffic and has certain commercial value and is located in the middle of the user pyramid. The PGC content at the top of the pyramid is well produced and requires a special production team, which has high requirements on capital, specialty, and technology and has extremely high commercial value. With the rapid development of short videos, content producers who have accumulated certain funds and experience have begun to change from personalization to organization. Some well-developed short video companies have signed up many potential content producers to help them grow, develop, and realize their business.

\subsection{Dissemination Content of Short Video Applications.} The competition of new media is fierce, and the pursuit of timeliness and accuracy of news is getting higher and higher. At this time, artificial intelligence can be an effective helper. Artificial intelligence can quickly search and sort out the required materials, check grammar and typos, and even analyze the authenticity and legitimacy of content through data logic. In the era of intelligent media, with sensors as the carrier and big data processing technology as the support, news sources are enriched and optimized, while intelligent robots undertake the task of collecting and sorting news clues.

In the Internet era, the threshold for content production is getting lower and lower, and users' demand for self-expression has been released. The era of nationwide content production is coming. At the same time, due to the impact of 
new media on traditional media, the pursuit of short video by capital, and the positive support strategy of Internet giants, outstanding talents from traditional media have poured into the wave of short video content entrepreneurship. Therefore, the short video industry has ushered in a golden period of content start-up, which has to show a rapid development in both the quantity and quality of content. Due to the different positioning of short video app, there are different degrees of differences between content, but it is still mainly concentrated in the scope of pan-entertainment. Figure 7 shows the vertical segmentation of short video industry.

Entertainment is not the long-term driving force for the development of short videos. Only truly nutritious content can bring about the long-term development of short videos as a carrier. For example, the content of Papi sauce reflects the current problems and arouses the sympathy of netizens under the funny shell. Rich and high-quality content is the fundamental driving force for short video platforms to attract users. The core competitiveness of content will be highlighted in the short video industry. In order to create more high-quality content, short videos begin to go deep into the vertical entertainment field from pan-entertainment. The content in the vertical field is increasingly rich and detailed, and the content value and commercial value in the vertical field are highlighted. The reason why the vertical field has become the development direction of short video content is mainly because of the following. First, the vertical short video content on the market is still at an early stage, and there is huge growth space for both the user scale and the audience. Second, capital supports the ecology of short video content, enabling more content creators in vertical fields to provide high-quality short video content. The third is that consumers will have more demand for the content of vertically subdivided fields under the background of consumption upgrading after forming the consumption habit of short videos.

\subsection{Communication Channels for Short Video Applications.} Communication channels of short video applications can be divided into internal channels and external channels of short video applications. Internal channels are mainly in the form of forwarding, comment, and barrage. External channels are more diverse and can be roughly classified into five categories: online video channels, information clients, platforms, short video channels, and vertical channels. Online video channels are usually checked by website editors, and the amount of broadcast of such channels is mainly obtained through user search and editing recommendations. Subjective factors such as Sohu Video, Youku Video, iQiyi, Tencent Video, Baidu Video, Bilibili, popcorn, and other platforms have a great impact on the video playback volume.

If short video content gets a high-quality recommended position, it will bring about a significant increase in short video playback volume. For example, some micromovies will advertise in various channels to obtain potential viewers after they go online, and these people will actively search for this micromovie on the corresponding websites. Most of the

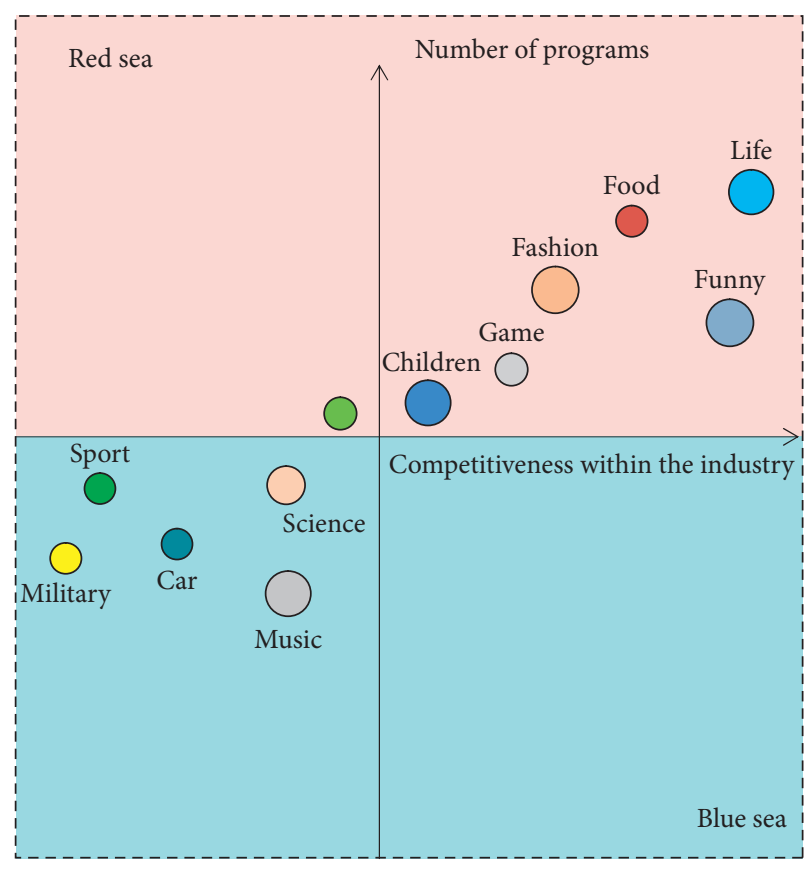

Figure 7: Short video industry vertical segmentation.

information client channels get the video playing volume through the platform's recommendation algorithm, for example, today's headlines, daily express, a little information, Netease news client, and UC browser. All use this recommendation algorithm mechanism to give videos corresponding labels and recommend them to corresponding user groups. At present, this recommendation mechanism is applied to many platforms, such as netease cloud music intelligent recommendation songs and Taobao intelligent recommendation products, which is also one of the future development trends.

In order to implement the decision-making of the decision-making level, it is necessary to have a management level to manage the daily work and an executive maintenance layer responsible for the implementation of safety plans and decisions. In this way, an information security organization with a hierarchical structure directly led by the $\mathrm{CIO}$ is formed. The security organization includes organizational decision-making layer, management control layer, and executive maintenance layer, as shown in Figure 8.

Platforms such as WeChat, Weibo, and QQ are convenient to meet more people with the same interests. Channels are important positions for short video applications to compete with each other, and the important significance of channels is not only to spread short video but also to facilitate users to search for corresponding short video content on the Internet. Some short video channels started from live broadcast platforms and were born as derivatives. However, since 2014, many people have realized that short video has more development prospects than live broadcast. More and more short video platforms have begun to appear in the public's view. "Second Shot" was upgraded to version 4.0 on July 28 , 2014, and the "Beauty shot" was also launched in 2014, which took only nine months for the number of users to exceed 100 million, as well as 


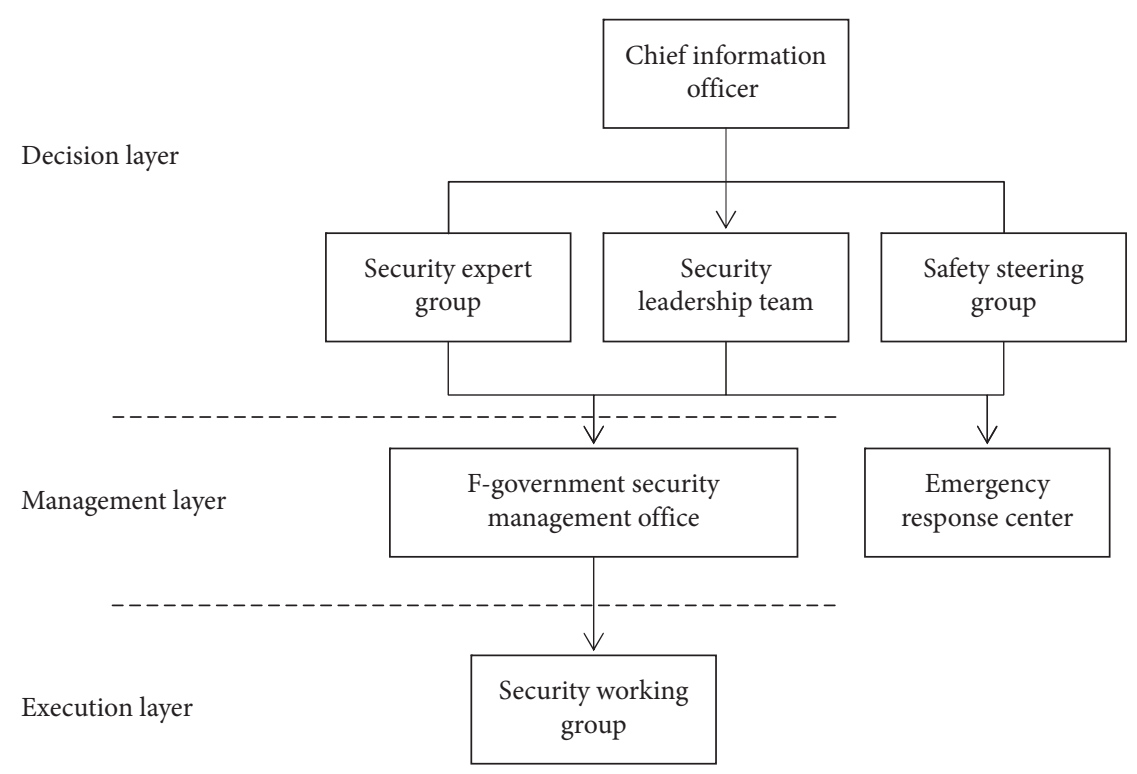

FIGURE 8: Hierarchical information security organization.

the earliest small video that can be made on the mobile side, pear video featuring short information video, and watermelon video split from the headlines.

The emergence of these short video channels cannot explain that short video is a trend rather than just a tuyere. What can really verify the correctness of this view is the emergence of vertical channels. E-commerce platforms represented by Taobao, Mushroom Street, Gift Talk, and so on can help users to understand commodities more comprehensively and thus promote the value-added of sales volume through short video. In addition, offline communication channels are also increasingly being paid attention to by short videos. Beauty shot and tremolo have expanded many offline communication channels through cross-border cooperation and achieved good results.

5.4. Typical Propagation Mode of Short Video App. The study of communication mode has always been the focus of communication studies. From the early one-way linear mode to the later two-way circulation mode, the communication mode has been continuously updated and developed with the change of people's communication mode. The popularity of app in the mobile Internet era has brought people new ways of information dissemination and challenged the traditional mode of dissemination. Users have replaced "communicators" and "audiences."

Personalized expression of users has replaced the herd mentality of users. Short video app, a new information carrier with attributes and short video forms, has brought subversive challenges to the traditional communication mode. Therefore, it is urgent to study new communication modes. Generally speaking, the communication modes of short video applications can be broadly divided into three types: UGC's information production mode, circular update transmission mode, and fission type information transmission mode. The low threshold and fast propagation of mobile applications make it possible for large-scale users to produce content. The emergence of media makes this UGC content can be shared and forwarded to each other. As long as the content is enough to attract attention, everyone can become a self-media. $4 \mathrm{~W}$ replaces $5 \mathrm{~W}$, Who (user), What (content), Why (motivation), and How (generation mode). UGC's content production mode mainly reflects the change of user status.

Circular update propagation mode refers to the user recreating the original video content once, twice, three times $\ldots n$ times through barrage, commenting, forwarding, and other forms to form an infinite circular update mode, which reflects the infinite content creativity of short video application. In addition to the continuous updating and recreation of content, short video applications also have the characteristics of fission information dissemination. As a node of fission link, users assume the dual roles of transmitter and receiver and play their own influence and role in the network structure of dissemination.

5.5. Suggestions on Optimizing the Dissemination Effect of Short Video App. Artificial intelligence helps new media people to process data, while a large amount of data generated by new media leads artificial intelligence to further grow in this environment, thus promoting the continuous development of new media itself. Under the algorithm mechanism, news can be produced as long as there are data, templates, and precise processes, and people's subjectivity and initiative are ignored [31]. The news produced often gives people the feeling of sameness and deja vu. The artificial intelligence algorithm in the new media solves the overload crisis of the traditional information dissemination paradigm in the Internet era and greatly improves the work efficiency of information production, distribution, and management.

According to the imitation theory of communication content, it is easy for people to obtain attractive behavior 
pattern information in mass communication content, and the content of media shows almost all conceivable behavior patterns. Imitation of media behavior mainly goes through three stages: (1) audiences pay attention to the behavior of characters described in media content; (2) audiences believe that this kind of behavior is attractive or effective and recognize or even imitate this kind of behavior. When the audience encounters similar situations in their own activities, they will imitate the behavior learned from the media. In order to get good communication effect, we need to optimize the source of communication content.

A high-quality content production system: Short videos are filled with a large amount of pan-entertainment content to meet the entertainment needs of the people. However, the deliberate creation and dissemination of "three customs" in order to get on the hot search become popular overnight, and attract eyeballs have affected the dissemination environment of the entire short video. In the content analysis of short video applications mentioned above, it is found that vulgar and illegal content only accounts for a small part of the content created by the whole user, and there are many high-quality and interesting content such as imparting life skills. To create a high-quality content, ecosystem requires the joint efforts of relevant government departments, short video platforms, and short video users. Relevant government departments have made clear in advance the illegal and illegal video content, and the communication platform will restrict the upload and display of such content. The short video platform of government departments needs to use correct values to guide the algorithm. The algorithm will be improved, and personalized positive energy works that are more in line with users' interests will be preferentially recommended to enlarge the influence and appeal of excellent works. Producers of head content such as "PAPI sauce" produce short video content with positive energy and high interest, which plays an exemplary and leading role. Vulgar content can only be popular for a while, gaining the attention of users for a while. What is really going on for a long time and is widely disseminated is high-quality positive energy content.

Communication pioneer Levin, who improved the video content auditing system, put forward the concept of "gatekeeper" in "Channels of Group Life." Information dissemination is always carried out according to specific communication channels. The opinions and characteristics of "gatekeeper" directly determine whether information dissemination is smooth or not. The mobile Internet era has impacted the "gatekeeper" theory. Some people even think that the "gatekeeper" no longer exists. In fact, the "gatekeeper" still exists, but the gatekeeper is more diversified. The government, media, platform vendors, and users are all acting as "gatekeepers." In order to establish a clean and healthy short video community environment, the government, media, platform vendors, and users should all play the role of "gatekeepers." The government promotes "real name system on the Internet" and implements credit system management for short video users. The platform side pushes positive energy content through technical algorithms and removes vulgar, violent, and pornographic short video content. CCTV and other mainstream media always pay attention to and report the chaos of short video content and urge them to correct it. Netizens actively complained and reported bad short video content, and multiple subjects made efforts to jointly improve the short video content auditing system.

Enhance user media literacy: In the mobile era, users have replaced the role of "gatekeeper" of traditional media and the role of "audience" who passively receive information, playing the dual roles of content producer and content receiver. Users upload vulgar, violent, and illegal short video content, and more users promote it. This is the source of chaos in short video applications, which is also an unavoidable problem in the early development of short video, a new content bearing form. From the source of governance, we need to improve the media literacy and self-discipline awareness of short video users and guide users to use media discourse rationally. Short video is already an important place for many users to express themselves and release themselves. It will be a progressive process for human beings to endow machine hardware with self-consciousness. Machine learning studies how computers simulate or realize human learning behaviors in order to acquire new knowledge or skills, reorganize existing knowledge structure, and continuously improve its performance. In the field of news media communication, the source code and working principle of the algorithm are encapsulated in the model framework to form a black box for users or for the audience to be calculated by the algorithm. The new media environment is increasingly complex. New media people must keep learning in order to keep up with the rapid changes in the overall new media environment. What is more, the software and hardware machines that meet the multiple needs of new media people and audiences can improve the accuracy of the algorithm, improve the algorithm, and quickly meet the application needs of the development of new media at low cost and promote the development of new media. In the era of intelligence, algorithm is power. Artificial intelligence technology not only highlights its advantages and strength in the field of new media but also penetrates into all aspects of human production and life. The algorithm is political, and it cannot achieve technology neutrality. It has begun to dominate the direction and content of manual selection in the field of new media.

\section{Conclusion}

Video content analysis is a very popular and important work in the field of machine learning and artificial intelligence. It is very difficult to analyze the video content produced by large-scale UGC, which is also the basis of subsequent video recommendation. At present, video analysis and classification methods are relatively few. Entertainment is not the long-term driving force for the development of short videos. Only truly nutritious content can bring about the long-term development of short videos as carriers. In this paper, topics such as pornographic picture identification, audio classification, and high-quality video classification are discussed, and methods such as machine learning, deep learning, and 
transfer learning are used. The rise of short videos is short, and the content uploaded by users shows geometric growth. However, the regulation of short videos market is still in the process of exploration, which inevitably leads to a series of problems such as the overflow of vulgar content, frequent infringement of users' privacy safety, and "anomie" of social members' behaviors. On the basis of studying the transmission mechanism of social short video applications, it is found that a series of chaos in social short video applications originates from the short video users who fail to perform the role of "gatekeeper" in the application process of social short video. Finally, the article puts forward countermeasures and suggestions from three aspects of forming the high-quality content production system, perfecting the short video content auditing system, and improving users' media literacy.

\section{Data Availability}

All the data used to support the findings of this study are included within the article.

\section{Conflicts of Interest}

The authors declare that they have no conflicts of interest.

\section{References}

[1] B. Becker, S. Holtgrefe, S. Jung et al., "Influence of the photoperiod on redox regulation and stress responses in Arabidopsis thaliana L. (Heynh.) plants under long- and short-day conditions," Planta, vol. 224, no. 2, pp. 380-393, 2006.

[2] C. J. Ferguson, "The influence of television and video game use on attention and school problems: a multivariate analysis with other risk factors controlled," Journal of Psychiatric Research, vol. 45, no. 6, pp. 808-813, 2010.

[3] P. Kucharczyk, S. Mohamed, and S. Münstermann, "On the influence of steel microstructure on short crack growth under cyclic loading," International Journal of Fatigue, vol. 41, pp. 83-89, 2012.

[4] S. Y. Davydov, "The effect of short-range repulsion of electronic shells on the energy spectrum of ions desorbed under the influence of electronic transitions: a relaxation model," Physics of the Solid State, vol. 39, no. 9, pp. 1498-1501, 1997.

[5] S. M. Vicente-Serrano and J. I. López-Moreno, "Differences in the non-stationary influence of the North Atlantic Oscillation on European precipitation under different scenarios of greenhouse gas concentrations," Geophysical Research Letters, vol. 35 , no. 18 , pp. $168-182,2008$.

[6] Y. Liu, L. Ou, C. Han, L. Zhang, and Y. Zhao, "The influence of $\mathrm{Mn}$ on the microstructure and mechanical properties of the Al-5Mg-Mn alloy solidified under near-rapid cooling," Journal of Materials Research, vol. 31, no. 8, pp. 1153-1162, 2016.

[7] Y.-L. Liang, X. Xing, H. Cheng et al., "SafeVchat: a system for obscene content detection in online video chat services," Acm Transactions on Internet Technology, vol. 12, no. 4, 2013.

[8] M. Wu, R. A. Joyce, H.-S. Wong, L. Guan, and S.-Y. Kung, "Dynamic resource allocation via video content and shortterm traffic statistics," IEEE Transactions on Multimedia, vol. 3, no. 2, pp. 186-199, 2001.

[9] W. Gao, Y. Tian, T. Huang, and Q. Yang, "Vlogging," Acm Computing Surveys, vol. 42, no. 4, pp. 1-57, 2010.
[10] F. Fund, S. A. Hosseini, and S. S. Panwar, "Under a cloud of uncertainty: legal questions affecting internet storage and transmission of copyright-protected video content," IEEE Network, vol. 30, no. 2, pp. 32-38, 2016.

[11] G. E. Boyhan, R. L. Torrance, J. Cook, C. Riner, and C. R. Hill, "Sowing date, transplanting date, and variety effect on transplanted short-day onion production," Horttechnology, vol. 19, no. 1, pp. 66-71, 2009.

[12] F. Turner, "Who controls the internet? Illusions of a borderless world," Technology and Culture, vol. 49, no. 1, pp. 296-297, 2007.

[13] K. Tarabanis, E. Kalampokis, and E. Tambouris, "Understanding the predictive power of social media," Internet Research, vol. 23, no. 5, pp. 544-559, 2013.

[14] C. Ouyang, M. La Rosa, A. H. M. Ter Hofstede, M. Dumas, and K. Shortland, "Toward web-scale workflows for film production," IEEE Internet Computing, vol. 12, no. 5, pp. 53-61, 2008.

[15] P. Salomoni, S. Mirri, and S. Ferretti, "A multimedia broker to support accessible and mobile learning through learning objects adaptation," Acm Transactions on Internet Technology, vol. 8, no. 2, pp. 1-23, 2008.

[16] M. Verhoeyen, J. De Vriendt, and D. De Vleeschauwer, "Optimizing for video storage networking with recommender systems," Bell Labs Technical Journal, vol. 16, no. 4, pp. 97-113, 2012.

[17] J. Yan, K. Katrinis, and M. May, "Media- and TCP-friendly congestion control for scalable video streams," IEEE Transactions on Multimedia, vol. 8, no. 2, pp. 196-206, 2006.

[18] H. Zheng and J. Boyce, "An improved UDP protocol for video transmission over Internet-to-wireless networks," IEEE Transactions on Multimedia, vol. 3, no. 3, pp. 356-365, 2001.

[19] X. Cheng, J. Liu, and C. Dale, "Understanding the characteristics of internet short video sharing: a YouTube-based measurement study," IEEE Transactions on Multimedia, vol. 15, no. 5, pp. 1184-1194, 2013.

[20] K. G. Kumar, J. S. Lipscomb, and A. Ramchandra, "The HotMedia architecture: progressive and interactive rich media for the Internet," IEEE Transactions on Multimedia, vol. 3, no. 2, pp. 253-267, 2001.

[21] X. Wang and H. Schulzrinne, "Incentive-compatible adaptation of Internet real-time multimedia," IEEE Journal on Selected Areas in Communications, vol. 23, no. 2, pp. 417-436, 2005.

[22] Q. Zhang, Z. Xiang, and W. Zhu, "Cost-based cache replacement and server selection for multimedia proxy across wireless internet," IEEE Transactions on Multimedia, vol. 6, no. 4, pp. 587-598, 2004.

[23] W. Cartwright, "New media and their application to the production of map products," Computers \& Geosciences, vol. 23, no. 4, pp. 447-456, 1997.

[24] F. Sardis, G. Mapp, J. Loo, M. Aiash, and A. Vinel, "On the investigation of cloud-based mobile media environments with service-populating and QoS-aware mechanisms," IEEE Transactions on Multimedia, vol. 15, no. 4, pp. 769-777, 2013.

[25] A. Derakhshan and H. Beigy, "Sentiment analysis on stock social media for stock price movement prediction," Engineering Applications of Artificial Intelligence, vol. 85, no. 10, pp. 569-578, 2019.

[26] D. Doty and M. Hajiaghayi, "Leaderless deterministic chemical reaction networks," Natural Computing, vol. 14, no. 2, pp. 213-223, 2015.

[27] C. L. Liu, Y. Q. Xu, H. Wu, S. S. Chen, and J. J. Guo, "Correlation and interaction visualization of altmetric 
indicators extracted from scholarly social network activities: dimensions and structure," Journal of Medical Internet Research, vol. 15, no. 11, p. e259, 2013.

[28] F. Gholami-Kordkheili, V. Wild, and D. Strech, "The impact of social media on medical professionalism: a systematic qualitative review of challenges and opportunities," Journal of Medical Internet Research, vol. 15, no. 8, p. e184, 2013.

[29] P. M. Archambault, "WikiBuild: a new application to support patient and health care professional involvement in the development of patient support tools," Journal of Medical Internet Research, vol. 13, no. 4, p. e114, 2011.

[30] A. Bernardi and M. Sintek, "Combining artificial intelligence, database technology, and hypermedia for intelligent fault recording," Clinical \& Experimental Metastasis, vol. 32, no. 4, pp. 383-391, 2015.

[31] A. Tommasel and D. Godoy, "Short-text feature construction and selection in social media data: a survey," Artificial Intelligence Review, vol. 49, no. 3, pp. 301-338, 2018. 